\title{
¿Cómo puede la universidad contribuir a construir un futuro mejor durante la pandemia de la COVID-19?
}

\author{
Fernando M. Reimers (D) \\ Universidad de Harvard, EE.UU.
}

Resumen. En este artículo se discute el impacto educativo de la COVID-19, destacando el papel de la universidad en promover innovación, para permitir no sólo mitigar los efectos negativos de la pandemia, sino reconstruir mejor. El autor argumenta que la integración de las funciones de extensión, investigación y educación de la universidad alineadas con la aspiración de reconstruir mejor, permitirán aumentar la relevancia de la universidad, como institución que faculte a la sociedad afrontar con éxito los desafíos super complejos creados por la pandemia.

Palabras clave: COVID-19; educación superior y desarrollo; supercomplejidad y educación superior; desafíos perversos y educación superior; innovación y educación superior.

Como a universidade pode contribuir para construir um futuro melhor durante a pandemia da COVID-19?

Resumo. Discute-se neste artigo o impacto educativo do COVID-19, destacando o papel da universidade ao promover inovação para permitir não só mitigar os efeitos negativos da pandemia, mas também reconstruir melhor. O autor argumenta que a integração das funções de extensão, pesquisa e educativas da universidade alinhadas com a aspiração de reconstruir melhor permitirão aumentar a relevância da universidade como instituição que permita à sociedade enfrentar com sucesso os desafios supercomplexos criados pela pandemia.

Palavras-chave: COVID-19; educação superior e desenvolvimento; supercomplexidade e educação superior; desafios perversos e educação superior; inovação e educação superior.

How can the university contribute to building a better future during the COVID-19 pandemic? Abstract. This article examines the educational impact of COVID-19, highlighting the role of the university in promoting innovation that contributes to not just mitigate the negative effects of the pandemic but to build back better. The author argues that the integration of the university's outreach, research and educational functions, aligned with the aspiration to build back better, will make it possible to increase the relevance of the university, as an institution that helps society face successfully the super complex challenges created by the pandemic.

Keywords: COVID-19; social embeddedness of universities; impact of higher education; university mission; universities as learning organizations; building back better and COVID-19; higher education and development, supercomplexity and higher education, wicked challenges and higher education, innovation and higher education.

\section{Introducción}

La universidad debe cumplir un papel fundamental ofreciendo una respuesta educativa a la pandemia de la COVID-19, no solamente sobrellevando las limitaciones que la misma ha creado para llevar a cabo las actividades propias de la universidad, por ejemplo, enseñar, sino promoviendo innovaciones esenciales para "reconstruir mejor" a través de actividades de investigación, enseñanza y vinculación social. Asumir este papel podría significar la transformación de la universidad misma, de manera similar a otras transformaciones que experimentó anteriormente, tales como el mayor énfasis en las funciones de extensión que fueron adoptadas por las universidades latinoamericanas después de la reforma de Córdoba, Argentina en 1919; o, la democratización en el acceso que adoptaron las universidades en Estados Unidos y en el resto del mundo como resultado de la segunda ola de democratización tras la segunda Guerra Mundial y de la tercera ola de democratización de los años setenta (Huntington, 1993). 
Esta oportunidad que la pandemia ofrece para reinventar la universidad ha sido caracterizada por Vivek Goel, rector de la Universidad de Waterloo como una oportunidad única en un siglo:

A medida que el mundo reabre, contra un telón de fondo de cambio acelerado y de desigualdades exacerbadas, las universidades deben aprovechar esta oportunidad de reinventarse única en un siglo.

Una universidad exitosa postpandemia será la que reconozca la oportunidad que ofrece este momento: la de aprovechar los desarrollos tecnológicos para construir sobre siglos de experticia; la de utilizar estas tecnologías como herramientas para romper las barreras al acceso; y la de comprender de qué forma están conectados estos desafíos (Goel, 2021).

La necesidad de aprovechar esta oportunidad histórica se deriva no solo de la urgencia derivada de los desafíos creados por la pandemia, sino de los retos precedentes que confrontaba la universidad, quizás por el agotamiento del sentido que animaba su misión, resultado éste de la última transformación significativa al término de la segunda guerra mundial que democratizó el acceso. Entre los retos precedentes se encuentran los cambios en la composición sociodemográfica de la matrícula universitaria, resultantes de la disminución del número de aspirantes tradicionales a la educación superior, producto de la transición demográfica, junto con el aumento del número de aspirantes procedentes de grupos sociodemográficos más vulnerables, resultado del éxito de los sistemas de educación secundaria en graduar un mayor porcentaje de alumnos de cada grupo de edad, en un contexto de reducción total del crecimiento del número de estudiantes del grupo de edad tradicional como resultado de la transición demográfica. En América Latina, entre los años 2000 y 2013, el porcentaje de estudiantes universitarios procedentes del $40 \%$ de la población con menos ingresos aumentó de 10.6\% a 16.8\% (Ferreyra et al. 2017).

A estos cambios demográficos se agrega una mayor expectativa de vida en la población, junto con el aumento en la necesidad de formación profesional continua, resultado de los cambios ocupacionales derivados de la incorporación de nuevas tecnologías en los procesos laborales.

Estos cambios demográficos y económicos generaron una necesidad de innovación en materia de desarrollo de nuevas competencias, personalización, apoyo a estudiantes no tradicionales para aumentar el porcentaje de alumnos que completaran exitosamente sus estudios (menos de la mitad lo hace), y nueva oferta de formación más allá de la formación tradicional en programas de titulación. Además, los aumentos en los costos de la educación superior crearon la necesidad de innovaciones que permitieran controlar y reducir costos y aumentar la flexibilidad de la oferta para satisfacer las necesidades de una población estudiantil más diversa.

La búsqueda de respuestas a estos desafíos, ya en los años anteriores a la pandemia, animó una serie de innovaciones en el sector de la educación superior. Por ejemplo, en los Estados Unidos, la Universidad Estatal de Arizona, una de las más innovadoras en la actualidad, desarrolló un nuevo modelo de universidad, bajo el liderazgo del rector Michael Crow. En el plazo de una década la universidad se reinventó para lograr una mejor integración entre la generación de descubrimientos y conocimientos, una mayor inclusión de grupos previamente excluidos y un mayor impacto 
social. Para hacerlo, aceleró un proceso de cambio institucional que reconceptualizó a la universidad como una "empresa de conocimiento" comprometida con el descubrimiento, la creatividad y la innovación, accesible a una base más amplia y diversa de estudiantes, tanto socioeconómica como intelectualmente (Crow y Dabars, 2015).

Esta misma necesidad de innovación había sido también expresada, antes de la pandemia, en referencia a las universidades en América Latina. Un análisis de las universidades en Latinoamérica hace un llamado a que se ocupen más intencionalmente de los desafíos que confrontan las sociedades, contribuyendo a promover la justicia y la igualdad, para lo cual serían necesarias innovaciones en materia de organización y gestión universitaria (Fernández-Lamarra, 2012).

Un informe reciente sobre educación y productividad en Iberoamérica enfatiza también la importancia de que la universidad y la educación técnica, contribuyan de forma más directa al aumento de la productividad económica (Jabonero, Iglesias y García, 2020).

A estos llamados a la reinvención de la universidad, en respuesta a los retos anteriores a la pandemia, agudizados algunos de ellos por la pandemia misma, se suman aquellos resultantes directamente de la pandemia: crisis social, económica y de gobernanza, mayores brechas en educación, nuevas necesidades de investigación. Además, la pandemia no ha concluido aún, ni está claro si tendrá una conclusión en un futuro cercano debido a las dificultades en distribución de vacunas a nivel global, y al logro de la vacunación efectiva de un porcentaje significativo de la población, sin lo cual, la prolongación de la pandemia facilita la aparición de nuevas cepas del virus, que resultan en nuevos brotes que continúan disrumpiendo el regular funcionamiento de las instituciones, como es el caso actualmente con la cepa Delta del coronavirus.

Este es el segundo de dos monográficos de la Revista Iberoamericana de Educación dedicados al estudio del impacto de la pandemia de la COVID-19 en educación. Los artículos publicados, seleccionados entre los 150 artículos que fueron presentados en respuesta a la convocatoria, dan cuenta del profundo impacto negativo educativo de la pandemia, en particular, como resultado de la prolongada suspensión de la educación presencial en muchas instituciones y países. Ello ha generado una crisis educativa profunda, seguramente la más significativa en la historia de la humanidad (Reimers, 2021a). El primer monográfico incluyó artículos referidos todos al impacto en la enseñanza básica. Este segundo monográfico contiene artículos referidos al impacto de la pandemia en la educación universitaria, además de otros que dan cuenta del impacto en otros niveles educativos.

Atender la crisis educativa causada por la COVID-19 requerirá, más que intentar restablecer las condiciones de funcionamiento de las instituciones y de los sistemas educativos anteriores a la pandemia, algo que aún no ha sido posible hacer en la mayoría de las escuelas y universidades de Iberoamérica. Para ello, será necesario "reconstruir mejor", utilizando la expresión con la cual el secretario general de las Naciones Unidas ha caracterizado la respuesta de la organización a la pandemia:

Para reconstruir mejor es necesario transformar el modelo de desarrollo de América Latina y el Caribe. 
En una región en la que los niveles de desigualdad se han vuelto ya insostenibles, ello supone desarrollar sistemas integrales de bienestar social accesibles para todas las personas.

Esto implica crear sistemas tributarios más justos, promover la creación de empleos decentes, fortalecer la sostenibilidad ambiental y reforzar los mecanismos de protección social.

También conlleva una mayor integración económica regional.

Y supone que las mujeres participen plenamente y en condiciones seguras en la vida pública y económica.

Por último, para reconstruir mejor, hay que reforzar la gobernanza democrática, la protección de los derechos humanos y el estado de derecho. En un momento en el que demasiados ciudadanos y ciudadanas se sienten excluidos, la rendición de cuentas y la transparencia son fundamentales. (Guterres, 2020).

Esta ambiciosa caracterización de las respuestas que serán necesarias para responder a la pandemia revela las múltiples y profundas dificultades que esta misma ha creado en diversos ordenes en las sociedades, las cuales han sido caracterizadas por la Organización de Naciones Unidas de la siguiente manera:

La pandemia es más que una crisis de salud; es una crisis económica, una crisis humanitaria, una crisis de seguridad y una crisis de derechos humanos, que ha afectado a las personas, las familias y las sociedades. La crisis ha puesto de relieve las fragilidades dentro de las naciones y entre ellas. No es exagerado sugerir que nuestra respuesta implicará rehacer y desimaginar las estructuras mismas de las sociedades y las formas en que los países cooperan para el bien común. Para salir de esta crisis será necesario un enfoque que abarque a toda la sociedad, todos los gobiernos y todo el mundo, impulsado por la compasión y la solidaridad (Naciones Unidas, 2020a, p.1).

En el caso de América Latina, las dificultades están bien sintetizadas en un informe de Naciones Unidas:

Partes de América Latina y el Caribe se han convertido en zonas críticas de la pandemia de COVID-19, exacerbada por estructuras de protección social débiles, sistemas de salud fragmentados y profundas desigualdades. El COVID19 provocará en la región la peor recesión de los últimos 100 años y se estima que generará una contracción del 9,1\% del producto interno bruto (PIB) regional en 2020. Esto podría aumentar el número de personas en situación de pobreza en América Latina en 45 millones (hasta llegar a un total de 230 millones de personas) y el número de personas en situación de extrema pobreza en 28 millones (llegando a un total de 96 millones de personas), poniéndolas en riesgo de desnutrición. En una región que experimentó un número significativo de crisis políticas y protestas en 2019, el aumento de las desigualdades, la exclusión y la discriminación en el contexto de la pandemia afectará negativamente el goce de los derechos humanos y los avances democráticos; situación que, de no atenderse, podría eventualmente derivar en malestar social y disturbios. (Naciones Unidas, 2020b).

La pandemia y su impacto constituyen un verdadero desafío adaptativo, un desafío de gran complejidad sin solución simple, un desafío que requerirá del mejor talento y de conocimiento basado en investigación científica, así como de capacidades individuales y colectivas para afrontarlo debidamente. En buena parte, el enorme sufrimiento causado por la pandemia es producto no solo de la pandemia misma, sino 
de las deficientes capacidades humanas con las que algunos líderes y ciudadanos respondieron a la crisis, de la toma de decisiones de política pública ignorando la mejor evidencia científica, así como de las debilidades de múltiples instituciones que amplificaron las pérdidas causadas por la pandemia, resultando en más de 200 millones de personas infectadas y más de 4.25 millones de fallecidos en el mundo.

En educación, por ejemplo, las deficiencias en infraestructura tecnológica, conectividad, y preparación de maestros y de estudiantes para aprender a distancia, significaron que los cierres de las escuelas se tradujeran en pérdidas de oportunidades de aprendizaje sin precedentes. El contraste entre los países con menor capacidad y preparación en esta área como los de América Latina, ante aquellos con mayor capacidad y preparación como Finlandia, Japón o Singapur, resultó en el mayor aumento de brechas de oportunidad educativa en América Latina, al ser la región del mundo con cierres de escuelas más extensas respecto a otros países (Reimers, 2021a).

Un informe reciente de un grupo experto encargado por el G-20 enfatiza esta falta de preparación social para una pandemia, e insta a la preparación para futuras pandemias:

La ampliación de la preparación para una pandemia no puede esperar hasta que termine la COVID-19. La amenaza de pandemias futuras ya está con nosotros. El mundo se enfrenta al peligro claro y presente de brotes de enfermedades infecciosas más frecuentes y letales. La pandemia actual no fue un evento de cisne negro. De hecho, en última instancia, puede verse como un ensayo general para la próxima pandemia, que podría ocurrir en cualquier momento, en la próxima década o incluso en el próximo año, y podría ser aún más dañina para la seguridad humana. (G-20 High Level Independent Panel, 1).

También es evidente que en las naciones donde los líderes políticos lograron una mejor coordinación con las autoridades sanitarias, donde la población siguió las recomendaciones de dichas autoridades, y donde hubo mayor capacidad institucional en el área de la salud pública, el impacto sanitario de la crisis fue menor, a diferencia de los países donde no hubo dicha coordinación o la misma capacidad institucional, o donde la población no siguió las recomendaciones basadas en evidencia científica. Igualmente, el impacto educativo de la crisis sanitaria ha estado mediado por las capacidades de las autoridades políticas y educativas, así como por la capacidad institucional educativa y por la respuesta de la población. Estas fragilidades institucionales en diversas áreas, tales como salud, educativa o sistemas de protección social, se refuerzan mutuamente. Las pérdidas de oportunidad educativa fueron así mayores no solamente en los países con infraestructuras educativas más deficientes, sino en aquellos con infraestructuras sanitarias más deficientes y con sistemas de protección social más deficientes. Este efecto combinado de múltiples formas de debilidad institucional ampliará por tanto las brechas entre los países con mayor y menor capacidad institucional. Es por ello, que la respuesta a la pandemia debe ser, como ha señalado la Organización de Naciones Unidas, multidimensional y sistémica, y es esta necesidad la que convierte al desafío de producir una respuesta en un desafío de supercomplejidad. Las universidades están idealmente situadas para atender a desafíos de supercomplejidad, como ha señalado Rafael Reif, rector del Instituto de Tecnología de Massachusetts, quien refiriéndose al cambio climático como un paradigma de desafío supercomplejo, lo caracteriza así: 
significa un problema social extremadamente complejo que no tiene una sola respuesta correcta ni una meta lineal clara, involucrando a múltiples partes interesadas con prioridades opuestas y donde no hay una autoridad central facultada para resolverlo (Reif, 2021, A8).

Las instituciones educativas, y la universidad en particular, tienen un papel fundamental que jugar en atender el desafío supercomplejo de la pandemia, porque la disponibilidad de conocimiento basado en evidencia científica y de adecuadas competencias humanas es esencial para avanzar en las cuatro dimensiones propuestas por Naciones Unidas como centrales en la respuesta a la crisis de la COVID-19:

- Dimensión social: basada en la igualdad y la protección social universal, independientemente del sexo o el género, la edad, la raza, el origen étnico, el idioma, la religión, la condición jurídica o el estatus migratorio, o de cualquier otra situación o circunstancia personal.

- Dimensión económica: basada en la creación de empleos decentes, sostenida por una mayor capacidad tecnológica local.

- Dimensión ambiental: basada en la protección de la naturaleza y el medio ambiente para las generaciones presentes y futuras.

- Dimensión política: basada en la democracia, el estado de derecho, la transparencia, la igualdad de género, la prevención de conflictos y la rendición de cuentas, así como en la participación y el acceso de la sociedad civil y las comunidades locales a la información en todas las fases de la formulación, la aplicación y la evaluación de las políticas públicas. (Naciones Unidas 2020b).

La presencia, o la ausencia, de dicho conocimiento y de dichas competencias humanas en cada sociedad para avanzar en esas cuatro dimensiones refleja los logros de los sistemas educativos en el pasado. Sin embargo, dado que la tarea de "reconstruir mejor" se extenderá durante los próximos años, es fundamental que las universidades y los sistemas educativos potencien y continúen desarrollando dicho conocimiento y dichas capacidades.

En sintonía con la idea de que es necesario fortalecer la resiliencia de los sistemas educativos para enseñar mejor durante la presente y futuras pandemias, y para contribuir a "reconstruir mejor" en una reciente publicación de la Academia Internacional de Educación, propongo que la respuesta a la pandemia requiere atender a tres horizontes interrelacionados: sostener las oportunidades de aprendizaje durante la pandemia, recuperar las perdidas educativas que han ocurrido durante la pandemia, y desarrollar la resiliencia y la capacidad de las instituciones educativas y del sistema educativo de educar mejor durante esta pandemia, en pandemias futuras, y en el futuro (Reimers, 2021b).

Desarrollar la resiliencia y la capacidad de las instituciones educativas requiere, a su vez, tres tipos de acciones: desarrollar la capacidad de enseñar de forma híbrida; superar las deficiencias de las instituciones que ofrecían educación de baja calidad y pertinencia a muchos estudiantes, que llevaba a algunos de ellos a desertar de la escuela; y, finalmente, ofrecer a los estudiantes las oportunidades de desarrollar las capacidades necesarias para construir un mundo mejor. 
Para "reconstruir mejor" en educación serán necesarios recursos financieros, humanos, liderazgo e innovación. Las universidades pueden hacer una importante contribución para enfrentar los desafíos adaptativos generados por la pandemia, a reconstruir mejor, estimulando innovación en una diversidad de áreas a través de sus funciones de investigación, enseñanza y vinculación social.

Si bien, puede parecer paradójico proponer que la universidad haga más en un contexto donde los diversos impactos de la pandemia podrían haber reducido su capacidad, incluso de mantener los niveles de operación anteriores a la pandemia, es propio de la universidad, en tanto organización que aprende, el responder a cambios en su ambiente externo no solo para adaptarse a ellos, sino para contribuir a construir un futuro mejor.

Las universidades en América Latina están bien posicionadas para ejercer este papel de liderazgo en "reconstruir mejor", ya que están bien valoradas por la sociedad, lo cual se expresa en la creciente demanda de acceso a la universidad; en el crecimiento de la matrícula y del número de instituciones; $y$, en el creciente financiamiento público a la universidad, que deriva en un aumento en términos reales de la inversión pública del 53\% entre los años 2010 y 2017 (Unesco, 2020). Además, la sociedad tiene más confianza en la universidad que en otras instituciones sociales, el $70 \%$ de la población confía en las universidades (Unesco, 2020).

Desde la vinculación, la investigación y la enseñanza, y creando sinergias entre acciones en estas tres dimensiones de su misión, la universidad puede hacer importantes aportes a la innovación necesaria en muchas de las áreas relevantes como réplicas para superar la crisis, que el informe de Naciones Unidas sobre la respuesta del COVID-19 en América Latina tipifica así:

Recomendaciones a corto plazo:

- Considerar la exploración de mecanismos para proporcionar a todas las personas que viven en la pobreza ingresos básicos de emergencia. Esto puede incluir la posibilidad de proveer el equivalente al umbral nacional de pobreza.

- Debería garantizarse el pleno acceso de todos quienes lo necesiten a la asistencia económica y humanitaria y a los servicios básicos.

- Aplicar medidas dirigidas a preservar las aptitudes y las capacidades productivas y de gestión.

- La respuesta multilateral internacional inmediata debería ampliarse a los países de ingreso medio.

- Recomendaciones para reconstruir con igualdad.

- Promover sistemas de bienestar integrales.

- Promover políticas industriales y tecnológicas sostenibles (ODS 9).

- Reforzar los sistemas tributarios progresivos para fortalecer la movilización de recursos internos (ODS 17).

- Preservar los ecosistemas naturales terrestres y marítimos estratégicos al tiempo que se reducen las desigualdades territoriales.

- Promover la integración económica regional.

- Fortalecer la gobernanza democrática, el estado de derecho, la rendición de cuentas y la transparencia. 
- La sostenibilidad medioambiental, sustentada en la Agenda 2030, debería servir de base para relanzar la cooperación multilateral, en particular en los países en desarrollo." (ONU, 2020b).

\section{Promover la innovación desde la vinculación}

Que la vinculación es parte de la misión de la universidad se reconoce -al menos- desde que Guillermo Humboldt estableció la Universidad de Berlín en 1811, como una de las tres instituciones de la llustración, siendo las otras dos: la escuela pública y la democracia. Este aspecto de la misión universitaria, llamada en ocasiones también extensión o impacto social, se refiere a la transferencia de conocimientos, tecnologías, o servicios, con el propósito deliberado de impactar las comunidades de las que forma parte, por ejemplo, apoyando el desarrollo de industrias, o contribuyendo a la atención de necesidades sociales -por ejemplo, en materia de salud pública u ofreciendo cursos de extensión de diversa índole-. En general existe más consenso sobre qué se entiende por enseñanza e investigación, las otras dos misiones de la universidad, que sobre el tipo de actividades que comprende la vinculación. La Asociación Nacional de Universidades e Instituciones de Educación Superior de México define la vinculación así:

La vinculación es una actividad estratégica de las Instituciones de Educación Superior que contribuye significativamente a las tareas de formación integral de los estudiantes; la producción y transferencia de conocimientos socialmente útiles que aporten soluciones a los problemas más urgentes de la sociedad y que incidan en el bienestar social, el crecimiento económico y la preservación de la riqueza de los recursos naturales; y la transferencia de conocimientos a la sociedad, así como la difusión de la cultura, el arte y el deporte en la sociedad (ANUIES, 2021).

En su forma más básica, es posible concebir a la vinculación como tareas para educar a la sociedad en general, para capacitar a las personas, en particular a aquellas que no son estudiantes en los programas tradicionales de titulación de la universidad, para contribuir a mejorar sus propias circunstancias y las de las comunidades de las cuales forman parte. Esta definición incluiría tanto programas de alfabetización a poblaciones analfabetas ofrecidos por la universidad, como programas de salud pública a la población en general, como programas que eduquen a empresarios transfiriendo tecnologías que mejoren los procesos productivos.

De las tres misiones de la universidad, enseñanza, investigación y vinculación, es quizás esta última la que esta más directamente relacionada con el compromiso de la universidad con avanzar un orden democrático. Tal vez una explicación a la falta de consenso sobre que significa la vinculación está en que la universidad moderna, la que es una creación del proyecto de la educación, es como la democracia misma, otro de los resultados de la ilustración, no un estado acabado, sino un proceso en continua evolución.

Aumenta la complejidad de este proceso de continuo desarrollo de la idea de vinculación, y de polémica sobre la misma, el que la universidad moderna tuvo que transformar una institución más antigua, la universidad medieval, cuya misión era la transmisión de dogmas religiosos. En este sentido, la tarea de transformar una institución existente es diferente a la creación de una institución nueva, como fue el caso 
de la creación de la educación pública, una institución creada al mismo tiempo que la universidad moderna, pero que surgió no para transformar una institución preexistente, sino para ocupar un vacío institucional. Por ello, no es casualidad que en los Estados Unidos los líderes de la revolución americana hayan dirigido su atención a la educación superior, antes que a la creación de escuelas públicas. Benjamín Franklin fundó la primera sociedad científica, la Sociedad Filosófica Estadounidense. John Adams establecería la segunda, la Academia Estadounidense de Artes y Ciencias, encargándola de utilizar el poder de las artes y las ciencias para la mejora social y la educación del público; Adams también dedicaría los primeros artículos de la constitución de Massachusetts a la educación superior, definiendo lo que debería enseñar para contribuir al progreso social. Franklin también fue fundador de una universidad, con mucho énfasis en el conocimiento práctico. Y, Thomas Jefferson diseñó la Universidad de Virginia para permitir a los estudiantes la experiencia de una comunidad académica democrática y cultivar las capacidades de razonamiento científico y compromiso cívico.

Los primeros cinco artículos de la constitución de Massachusetts, que John Adams escribió en 1780, todos dedicados a la educación superior, articulan esta visión de una educación para la mejora social.

La sabiduría y el conocimiento, así como la virtud, deben difundirse de forma generalizada entre la población, siendo necesarios para la preservación de sus derechos y libertades; y como éstos dependen de la difusión de las oportunidades y ventajas de la educación en las diversas partes del país, y entre los diferentes órdenes del pueblo, será deber de los legisladores y magistrados, en todos los períodos futuros de este Estado, cuidar la intereses de la literatura y las ciencias, y todos sus seminarios; especialmente la universidad de Cambridge, las escuelas públicas y las escuelas de gramática de las ciudades; fomentar sociedades privadas e instituciones públicas, recompensas e inmunidades, para la promoción de la agricultura, las artes, las ciencias, el comercio, los oficios, las manufacturas y la historia natural del país; apoyar e inculcar los principios de humanidad y benevolencia general, caridad pública y privada, laboriosidad y frugalidad, honestidad y puntualidad en sus tratos; sinceridad, buen humor y todos los afectos sociales y sentimientos generosos entre la gente (Constitución de Massachusetts de 1780).

Francisco de Miranda, un apasionado de las ideas de la llustración y quien eventualmente sería uno de los líderes de la revolución por la independencia de Sudamérica, también tuvo gran interés en el papel de las universidades en promover un orden social de personas libres. Luego de que la Inquisición exigiera su arresto, siendo Miranda miembro del ejército español, a causa de sus intereses en las obras de los filósofos de la Ilustración, Miranda huyó a Estados Unidos en 1783, donde conoció a George Washington, Thomas Paine, Samuel Adams, Thomas Jefferson, Alexander Hamilton y otros líderes de la revolución americana. Durante su estadía en Estados Unidos se interesó especialmente en el papel de la legislación y en el de las diversas universidades de las colonias americanas, visitando Princeton, Yale y Harvard (Racine, 2002). Años después, en 1810, es Francisco de Miranda quien presentó a Andrés Bello y a Simón Bolívar al profesor Joseph Lancaster, quien había desarrollado un método para educar de forma eficiente a estudiantes pobres, cuando Bello y Bolívar, junto con Luis López Méndez, viajaron a Londres en busca de apoyo 
financiero para la gesta de la independencia. Como resultado de estos intercambios de ideas, Bolívar enviaría a dos maestros venezolanos a estudiar en la escuela que había creado Lancaster en Londres, y posteriormente invitaría al mismo Lancaster a visitar la escuela en Caracas, entre 1825 y 1827. Bello, por su parte, se convertiría en el primer rector de la Universidad de Chile, la primera universidad republicana de ese país, desde la cual demostraría y promovería el papel de los profesores universitarios como intelectuales públicos, participando en polémicas públicas sobre las instituciones de la nueva República. Entre estos, los debates de Andrés Bello con Domingo Faustino Sarmiento, fundador de la primera escuela de maestros en Chile, contribuyeron a la consolidación de la influencia de Sarmiento en la creación de la escuela pública. Es este un ejemplo del papel de la universidad, y de los universitarios y de las ideas, en la creación de la escuela pública, siendo otro ejemplo el desarrollo del proyecto de la Secretaría de Educación Pública de México por José Vasconcelos, rector de la Universidad Nacional, de cuya Secretaría pasaría Vasconcelos a ser el primer titular en julio de 1921 para iniciar un programa de transformación educativa animado por la aspiración de una profunda transformación social. La aspiración de Vasconcelos sobre la importancia de las tareas de vinculación de la universidad está claramente expresada en su discurso de nombramiento como rector de la Universidad Nacional en 1920, en el que dijo "Yo no vengo a trabajar por la Universidad, sino a pedir a la Universidad que trabaje por el pueblo".

En 1862, el presidente de Estados Unidos Abraham Lincoln firma la ley que autoriza el financiamiento de instituciones de educación superior, con la misión de promover el desarrollo de la agricultura, la ciencia, la ingeniería y las artes militares como respuesta a la revolución industrial y a los cambios sociales que esta generó. Esta ley es la base de la creación de las universidades estatales con vinculación a actividades productivas en los Estados Unidos; así como de universidades privadas, como la Universidad de Cornell y el Instituto de Tecnología de Massachusetts. Con el tiempo, esta misión de "extensión" de la universidad, se amplió para incluir una diversidad de acciones que permitieran a las universidades ser entidades que contribuyeran a mejorar las comunidades de las cuales formaban parte, universidades emprendedoras y socialmente arraigadas, abiertas a la sociedad, al cambio social e involucradas en generar impacto (Ramírez, 2020).

La reforma universitaria de 1918 en Córdoba, Argentina consolida esta aspiración de vinculación, de extensión, de la universidad latinoamericana, sirviendo la reforma argentina de ejemplo a reformas en otros países.

Diversos procesos nacionales profundizan esta vocación de la universidad de contribuir al desarrollo económico y social. En el caso de México, por ejemplo, los movimientos estudiantiles de 1968, y la respuesta del gobierno Federal a los mismos, motivan una serie de reformas a las leyes orgánicas que rigen el funcionamiento de las universidades estatales, a comienzos de los años setenta, estas nuevas leyes proponen la autonomía de las universidades estatales (académica, de gobierno, y presupuestaria) junto con mayor énfasis en las actividades de extensión.

En el caso de Brasil, la reforma universitaria de 1968 moderniza la universidad, creando la red de programas de postgrado que tuvieron un enorme impacto económico, social y político. La creación de los programas de ciencias sociales en 
Brasil establece la base para el desarrollo de la ciencia social contemporánea y de la democratización de Brasil-aun cuando, paradójicamente, dicha modernización ocurrió durante regímenes militares autoritarios-. Es desde la universidad, en Brasil, donde se fragua la preocupación por la creación de instituciones democráticas y la preocupación por la desigualdad y la justicia social como prioridades que deben ser atendidas en un marco democrático. Son estas reformas las que inician un creciente interés por la vinculación de las universidades, que producen diversas innovaciones institucionales. Por ejemplo en 1997, siendo presidente de Brasil Fernando Henrique Cardoso, uno de los profesores universitarios que lidera la creación de las ciencias sociales y del interés por la democracia en la universidad, y siendo ministro de educación Paulo Renato Souza, un exrector universitario, el Ministerio de Educación crea el Programa de Apoio à Avaliaçao Educacional, cuya misión es apoyar a grupos de investigación universitarios para hacer evaluación e investigación que apoye a la escuela pública. Cinco universidades crearon centros de vinculación con escuelas públicas como parte de este programa: la Universidad de Juiz de Fora, la Universidad de Belo Horizonte, la Universidad de Brasilia, la Pontificia Universidad Católica de Río y la Universidad Federal de Río de Janeiro.

Es precisamente esta vinculación con los sistemas educativos preuniversitarios una de las áreas donde la universidad puede promover innovación para "reconstruir mejor", algo que es en el interés de la universidad hacer, pues la deficiente preparación preuniversitaria de los estudiantes es una de las barreras a la calidad universitaria. Es esta una de las razones por la cual muchas universidades tienen sus propias instituciones preuniversitarias, o tienen vínculos con escuelas primarias y secundarias. El aumento considerable de la proporción de estudiantes universitarios en América Latina procedente de los grupos de menores ingresos, que en promedio han tenido acceso a una educación preuniversitaria de menor calidad, representa un nuevo desafío para la calidad universitaria y por ello una razón para que la universidad contribuya a mejorar la calidad de la educación en esos niveles, calidad que se vio seriamente amenazada durante la pandemia.

En un estudio reciente del papel de la universidad en la vinculación con instituciones educativas preuniversitarias durante la pandemia, encontramos que muchas universidades respondieron a la crisis creando alianzas con escuelas y sistemas educativos, resultando en una serie de innovaciones que contribuyeron a mantener las oportunidades educativas durante la fase más crítica de la pandemia (Reimers y Marmolejo, 2021). Estas colaboraciones generaron siete tipos de innovaciones:

1 Llevar a cabo investigación y análisis para apoyar a los tomadores de decisiones en la formulación de estrategias de continuidad educativa (extensión e investigación).

2 Crear conocimiento basado en la investigación en las escuelas en el contexto de la pandemia (investigación).

3 Crear recursos educativos y tecnológicos y plataformas en línea para estudiantes y maestros, incluidos los esfuerzos para apoyar la conectividad (extensión y docencia).

4 Llevar a cabo desarrollo profesional para maestros, administradores de educación y padres (extensión). 
5 Destacar la importancia de la atención al apoyo socioemocional a los estudiantes (extensión).

6 Aprendizaje e innovación organizacional (sinergias entre investigación, docencia y extensión).

7 Desarrollar innovaciones en la docencia: involucrar a los estudiantes universitarios en estas colaboraciones con las escuelas (docencia). (Reimers y Marmolejo, 2021).

Estas colaboraciones y las innovaciones que las mismas generaron, fueron facilitadas por una misión y una estrategia universitaria que promovían la vinculación, a través de la colaboración e integración en diversos esfuerzos dentro de las instituciones, y por estructuras y colaboraciones preexistentes con las escuelas. Por ejemplo, la universidad EAFIT en Colombia colaboró con el Ministerio, desarrollando la plataforma para la enseñanza digital, constituyendo la estrategia de enseñanza remota durante la pandemia. En Chile, el presidente de la República invitó a los rectores de las dos principales universidades del país, la Universidad de Chile y la Pontificia Universidad Católica de Chile, a conformar una mesa social para que colaborase con el Gobierno en desarrollar respuestas a la pandemia. En México, la Universidad de Guadalajara integró los múltiples campos de la universidad con las instituciones de educación preuniversitaria también de la universidad, en actividades de formación del personal para enseñar de forma remota. En Brasil, la Fundación Getulio Vargas colaboró con secretarios municipales de educación, apoyando el desarrollo de estrategias para enseñar durante la pandemia (Reimers y Marmolejo, 2021).

Mas allá de su común origen como instituciones del proyecto democrático de la Ilustración, es comprensible esta vinculación de la universidad con sistemas educativos preuniversitarios. Como institución especializada en el aprendizaje y en la enseñanza, la universidad tiene capacidades sobre estos temas que son de valor para las instituciones educativas en niveles preuniversitarios. Por ejemplo, típicamente las universidades tienen más experiencia en enseñanza a distancia que las instituciones preuniversitarias, en 2018, uno de cada cinco estudiantes universitarios en Latinoamérica estudiaba a distancia, y el número de estudiantes universitarios latinoamericanos estudiando a distancia se duplicó entre 2010 y 2018 (Unesco, 2020). Aquellas universidades con dicha experiencia en educación a distancia pudieron ofrecer tanto capacitación como plataformas tecnológicas a niveles preuniversitarios durante la pandemia.

De parte de los sistemas educativos, la vinculación con la universidad tiene a su vez sentido porque existe allí una reserva de capacidad intelectual de enorme valor para apoyar la educación preuniversitaria. Tradicionalmente, las universidades tienen más recursos intelectuales y mayor infraestructura que las escuelas y, por ello, ante la necesidad de enfrentar un desafío supercomplejo como el creado por la pandemia, es comprensible que las escuelas buscasen asociarse con instituciones que pudiesen apoyarlas. Muchas de las actividades que he identificado como esenciales en el proceso de aumentar la resiliencia de los sistemas educativos y de aumentar su capacidad de reconstruir mejor durante la pandemia (Reimers, 2021b), son actividades a las cuales la universidad puede aportar, estas son: 
I. Evaluar tanto como ha cambiado el contexto, así como las necesidades que estos cambios han creado:

1 Evaluar el bienestar de los estudiantes y su disposición para aprender.

2 Evaluar el acceso y la participación de los estudiantes.

3 Evaluar el bienestar y disposición de maestros a enseñar.

4 Evaluar los cambios en el contexto, el impacto de la pandemia en las comunidades.

5 Evaluar el funcionamiento del sistema educativo.

II. Desarrollar una estrategia para enseñar durante la fase aguda de la pandemia o para recuperarse de ella:

1 Comprometerse a apoyar a todos los alumnos.

2 Desarrollar una plataforma educativa que integre la educación presencial con la enseñanza remota y que permita la personalización y la diferenciación.

3 Repriorizar el plan de estudios. Basarlo en el desarrollo de competencias y en la educación integral del niño.

4 Acelerar el aprendizaje y personalizar.

5 Apoyar la salud mental y el bienestar emocional de los estudiantes.

6 Evaluar la efectividad de las innovaciones que se han realizado.

7 Integrar servicios que apoyen a los estudiantes (salud, nutrición).

III. Incrementar la capacidad:

1 Desarrollar la capacidad de las escuelas.

2 Desarrollar la capacidad de profesores, lideres escolares y el personal escolar.

3 Crear alianzas entre escuelas y otras organizaciones.

4 Comunicarse con los padres y desarrollar habilidades parentales.

5 Construir redes escolares.

El lograr una mayor articulación entre la universidad y los sistemas educativos requerirá sobrepasar las barreras estructurales que existen para ello. Muchos sistemas educativos públicos están desconectados de las universidades, la formación docente tanto inicial como continua ocurre fuera de las universidades, las actividades de investigación y evaluación de sistemas educativos se llevan a cabo sin participación de las universidades. Igualmente, muchas universidades, incluidas en sus escuelas de educación, tienen una vinculación limitada con los sistemas educativos. Esto hace de singular valor el ejemplo que ofrecen iniciativas como el Programa de Apoio à Avaliação Educacional que adelantó el Ministerio de Educación de Brasil, mencionado anteriormente, y que ilustra de que forma la política pública y el financiamiento pueden crear las condiciones estructurales que faciliten dicha articulación. Las condiciones de emergencia creadas por la pandemia promovieron la innovación en la construcción de nuevas formas de articulación, pero la consolidación de las mismas requerirá de políticas publicas propicias y de recursos financieros.

Por supuesto, la contribución de la universidad a "reconstruir mejor" durante la pandemia a través de la vinculación, puede incluir el enlace con otras instituciones además de los sistemas educativos, tales como las empresas o las instituciones de salud. Un área obvia de vinculación concierne a la salud pública y la atención médica, 
dado que las mismas son áreas centrales en la crisis de la COVID-19. Numerosas universidades administran hospitales y centros de salud que ofrecen servicios a la comunidad. Por ejemplo, durante la pandemia, el Hospital Universitario de la Universidad Autónoma de Nuevo León en México ha ofrecido atención médica especializada a la comunidad. La Clínica comunitaria ha ofrecido pruebas diagnósticas y servido como centro de referencia para el diagnóstico. Además, la universidad ha organizado brigadas de aplicación de vacunas a estudiantes y trabajadores, atención psicológica a estudiantes y trabajadores y atención psicológica a la comunidad. También en México, la Universidad de Guadalajara estableció la "Sala de Situación en Salud", para coadyuvar en el diagnóstico y políticas sanitarias.

Otras áreas en las que la universidad puede contribuir a innovar desde la vinculación incluyen los desafíos del cambio climático, o el planeamiento urbano, o promover la innovación económica o social, o promover el bienestar de las personas a través de actividades culturales. Al igual que en el caso de la vinculación con instituciones educativas, una vinculación efectiva requerirá de políticas públicas y de condiciones estructurales que las apoyen. Como indicación de algunos de los desafíos en estos esfuerzos de vinculación, un estudio reciente sobre las universidades en América Latina concluye que son mayores los esfuerzos de la universidad por vincularse con las empresas, que la demanda de las empresas por dicha vinculación, la cual es relativamente baja (Unesco, 2020).

En síntesis, la crisis generada por la pandemia acelera la necesidad de la difusión de conocimientos basados en investigación científica, de saber experto. Por ello es esencial que la universidad contribuya a la creación de ecosistemas amplios e incluyentes de aprendizaje continuo, tanto a través de programas administrados directamente por cada universidad, como a través de programas que reflejen la colaboración entre varias instituciones, para que permita compartir los costos, aumentar la escala desde la cual crear dichos recursos y el alcance de los mismos.

\section{Promover la innovación desde la investigación}

Las universidades pueden contribuir a la esencial innovación para "reconstruir mejor" a través de la generación de conocimientos, una de sus misiones centrales. Por ejemplo, para continuar en el área de la enseñanza preuniversitaria, es que las universidades pueden realizar investigación sobre las innovaciones educativas que ocurrieron durante la pandemia, como una forma de mantener la continuidad educativa. Un estudio reciente de treinta y una innovaciones de este tipo demuestra que las mismas tienen importantes implicaciones para avanzar en la transformación de los sistemas educativos alineados con la ambiciosa propuesta de Futuros de la Educación propuesta por la Unesco (2021).

Estas treinta y una innovaciones desarrollaron formas eficaces de educar integralmente, de repriorizar el currículo, de utilizar tecnologías apropiadas para construir sistemas educativos híbridos que apoyasen más eficazmente a los estudiantes (Reimers y Opertti, 2021). Otros estudios confirman que las respuestas de las instituciones educativas a la pandemia generaron un dividendo de innovación (Reimers y Schleicher, 2020). 
Aprender de estas innovaciones es esencial por dos razones: en primer lugar, porque aportan claves al tipo de innovaciones que contribuirían a "reconstruir mejor"; $y$, en segundo lugar, para identificar las fortalezas en organizaciones y sistemas. Visibilizarlas y estudiarlas es una estrategia eficaz de transformación organizacional y social como demuestra el enfoque de investigación apreciativa ${ }^{1}$ (Cooperrider, Whitney y Stavros, 2004).

El conocimiento experto, basado en investigación científica, es la base de todas las dimensiones del proceso de "reconstruir mejor" que propone Naciones Unidas, sean estas: la creación de modelos de desarrollo más incluyentes y sustentables, promover la inclusión social y la reducción de la desigualdad, la creación de sistemas de bienestar social accesibles para todas las personas, el desarrollo de sistemas tributarios más justos, la creación de empleos, la sostenibilidad ambiental, la protección social, la integración económica, la igualdad de género, la profundización de la democracia, protección de derechos humanos y del estado de derecho; el descubrimiento de soluciones a estos desafíos requiere de conocimiento, una tarea para la cual la universidad esta singularmente calificada. No es de sorprender que muchas universidades hayan adoptado ya los objetivos de desarrollo sustentable acordados en la asamblea general de las Naciones Unidas en 2015, como un marco orientador de su actuar.

La misión de investigación en la universidad es de especial importancia en América Latina porque la mayor parte de la producción de conocimiento científico y tecnológico en esta región ocurre en las universidades, particularmente en las públicas (Unesco, 2020). En el estudio de vinculación de las universidades de América Latina ya reseñado, se estudia de forma particular la vinculación en el contexto de la pandemia de la COVID-19. El informe documenta una serie de iniciativas de universidades en la región orientadas a atender las necesidades sanitarias creadas por la pandemia, entre ellas: el desarrollo de un test molecular para diagnosticar la COVID-19 por las universidades de San Martín y de Quilmes en Argentina; el diseño de ventiladores pulmonares de bajo costo por la Pontificia Universidad Católica del Perú; el diseño de mascarillas de protección para el personal médico por la Universidad de San Carlos de Guatemala; la secuenciación del genoma de una cepa de la COVID-19 por la Universidad de San Francisco de Quito en Ecuador, para hacer seguimiento al virus; el desarrollo de un biosensor para detectar casos de virus de forma costo-efectiva en la Universidad Nacional Autónoma de México; la realización de test de diagnóstico en la Universidad de la Plata en Argentina; el desarrollo de una cabina sanitizadora en la Universidad de Chile; el desarrollo de un sistema de ventilación no invasivo en la Universidad Autónoma de Manizales en Colombia; el desarrollo de un dispositivo de ventilación mecánica en la Universidad de Concepción en Chile; el desarrollo de un test rápido para diagnosticar casos asintomáticos en la Pontificia Universidad Católica de Chile; el desarrollo de un desinfectante que elimina el virus de superficies en la Universidad de los Andes en Colombia; el desarrollo para una vacuna comestible contra el virus en la Universidad Autónoma de Nuevo León; la Universidad de Piura en Perú desarrolló cánulas de alto flujo de oxígeno para atender a casos extremos de infección; una vacuna nasal en espray desarrollada por la Universidad de San Pablo en Brasil; la Universidad Austral de Chile desarrolló una prueba de bajo costo

\footnotetext{
${ }^{1}$ En inglés Appreciative Inquiry
} 
para un diagnóstico masivo; la evaluación de fármacos existentes para atacar la enfermedad en la Universidad Nacional Autónoma de México; el desarrollo de un kit de diagnóstico molecular en la Universidad Mayor de San Simón de Bolivia; el desarrollo de un ventilador mecánico en la Universidad Simón Bolívar de Venezuela; en Uruguay se estableció el centro de Innovación en Vigilancia Epidemiologia (Unesco, 2020). Además de estos desarrollos tecnológicos, investigadores de universidades latinoamericanas publicaron más de 600 trabajos científicos en los primeros cuatro meses de la pandemia.

Es evidente que la pandemia estimuló la actividad de investigación en la Universidad y el desarrollo de tecnologías y que ello hizo visible, al resto de la sociedad, la importancia de dicho conocimiento y de la universidad. En palabras de Alejandro Gaviria, rector de la Universidad de Los Andes de Colombia:

una conclusión que uno puede sacar de la pandemia, del cambio climático, de lo que está ocurriendo en las democracias de buena parte del mundo, es que el conocimiento importa, que las ideas tienen un papel que cumplir.

Que de alguna manera no podemos dejarle las decisiones cruciales de nuestras sociedades, en un momento ominoso e inquietante, a los demagogos que nos gobiernan. Creo que ahí la universidad tiene que jugar un papel, y que tarde o temprano nuestro papel será más relevante del que ha sido históricamente (Gaviria, 2020).

Sería muy valioso poder identificar cuáles fueron las condiciones que posibilitaron la mayor productividad de investigación y de innovación, en los centros donde esto ocurrió, de cara a acelerar estos procesos en el futuro.

\section{Promover la innovación desde la enseñanza}

La educación es el tercer componente de la misión de la universidad. La pandemia creó, también para la universidad, la necesidad de encontrar formas efectivas de educar en contextos de distanciamiento social y de otras dificultades creadas por la crisis. Como respuesta a estos desafíos las universidades innovaron. Esta innovación incluyó tanto un énfasis en desarrollar competencias diferentes, así como formas alternativas de enseñanza. En la medida en que las universidades tuvieron que utilizar formas de educación remota, esto exigió mas responsabilidad de parte de los alumnos por organizar su propio aprendizaje. Además, la situación de estrés creada por la pandemia hizo que muchas instituciones dedicasen más atención al bienestar y desarrollo socioemocional de los estudiantes. Este énfasis en habilidades más allá de las cognitivas es importante para preparar a los estudiantes para un mundo en el cual dichas habilidades son necesarias para la participación económica y social. Un estudio de las habilidades que exigen los empleadores que contratan egresados de secundaria en cinco industrias: automotriz, motorista, hotelería, financiero y alimentos en Argentina, Brasil y Chile encontró que los empleadores expresaban mayor dificultad en encontrar candidatos con habilidades socioemocionales para realizar sus trabajos, que con habilidades cognitivas o técnicas específicas y que las habilidades que más valoraban eran las socioemocionales (Bassi, Busso, Urzua y Vargas, 2012). El mismo estudio, evaluó las habilidades de una muestra de jóvenes que habían alcanzado diversos niveles de escolaridad en Argentina y Chile, encontrando que, si bien hay un claro y significativo aumento en habilidades cognitivas a medida que aumenta el nivel 
de escolaridad, no se observan las mismas diferencias con relación a las habilidades metacognitivas, autoeficacia y habilidad social. En el caso de estas tres últimas, las mayores diferencias se observan entre los grupos de jóvenes que completaron solo la educación primaria con relación a quienes solo completaron la secundaria, pero las diferencias son inexistentes entre los grupos que han completado la secundaria, la educación técnica superior o la educación universitaria (Ibid). Resultados semejantes fueron encontrados en otro estudio que incluyó a personas entre 25 y 55 años en Bolivia y Colombia, encontrando que si bien quienes habían completado estudios universitarios tenían mayores destrezas cognitivas (comprensión lectora) que los que no habían ido a la universidad, las diferencias eran mucho menores en el caso de las capacidades no cognitivas (Ferreyra et al. 2017). Es decir, estos estudios no ofrecen evidencia de que la educación universitaria aumente las capacidades socioemocionales².

Seguramente las innovaciones que se llevaron a cabo en las universidades para enseñar a distancia durante la pandemia representaron la mayor inmersión en educación a distancia en la que participaron los profesores de las más de 10.000 instituciones de educación superior en América Latina (y en el resto del mundo). Esta inmersión se dio con niveles variables de infraestructura y de apoyo a los profesores. La misma representa una oportunidad única de aprender a partir de este dividendo de innovación. A esta oportunidad se refiere el rector Goel en la cita reseñada anteriormente cuando habla del momento creado por la pandemia como una oportunidad única para la reinvención de la universidad, donde se utilicen las tecnologías para romper barreras al acceso, para incluir a grupos previamente excluidos. Una tarea urgente para muchas universidades es estudiar que aprendieron sus profesores y sus administradores, como resultado de las innovaciones que realizaron, por necesidad, durante la pandemia y discernir cuales son las implicaciones pedagógicas y programáticas de estos aprendizajes.

Como fue señalado anteriormente, muchas universidades innovaron en materia pedagógica apoyando a escuelas y a sistemas preuniversitarios, en ocasiones estos esfuerzos fueron parte de esfuerzos por innovar para permitir la enseñanza remota en la propia universidad. Este fue el caso de los desarrollos de capacidades para enseñar remotamente en la Universidad de Guadalajara, México, ofrecidas simultáneamente a profesores universitarios y a los profesores de las secundarias superiores de la universidad. Igualmente, el Tecnológico de Monterrey desarrolló un modelo para educación en línea que fue utilizado para apoyar la enseñanza remota tanto en la universidad como en las secundarias superiores de la universidad. También en México, la Universidad de Nuevo León creó un modelo para educación en línea, 90 mil aulas híbridas, donando dispositivos móviles a estudiantes de bajos recursos, apoyando a escuelas que no tenían acceso a internet para que lo tuviesen, ofreciendo capacitación a profesores para educación en línea e hibrida, y creando aulas híbridas en dependencias académicas. En Brasil, la Universidad Federal de Juiz de Fora, basándose en relaciones sólidas de colaboración con sistemas públicos de educación

\footnotetext{
2 Debido a que estos son estudios correlacionales, y no experimentales, no permiten establecer inferencia causal en las diferencias observadas. Las diferencias entre los grupos comparados pueden deberse a efectos de la educación superior o a efectos de selección. Sin embargo, la ausencia de diferencias en habilidades socioemocionales entre grupos con estudios universitarios y otros, indica que es improbable que la educación superior desarrolle estas destrezas de forma significativa.
} 
en varios estados, apoyó la creación de una plataforma de enseñanza remota en el Estado de São Paulo y en otros estados brasileños y ofreció capacitación a profesores y directivos educativos.

Sin duda, innovaciones programáticas semejantes ocurrieron en muchas universidades en el mundo no solamente con relación a programas de titulación, sino con relación a modalidades de formación más flexibles, vía cursos cortos o certificados, que permitan la actualización continua profesional. Ello será de creciente importancia en América Latina en un contexto en el que la tecnología transforma los procesos de producción, eliminando la demanda laboral en algunas áreas, y creando demandas nuevas. Una síntesis reciente de estudios sobre el tema demuestra que un número considerable de empresas en el sector formal de la economía en Iberoamérica encuentran difícil contratar personal calificado de acuerdo con sus necesidades (Ríos, 2020). Otro informe reciente sobre educación superior y competitividad enfatiza la importancia de las competencias transversales, las cuales no son cultivadas en la mayoría de las universidades de Iberoamérica, aun cuando las autoridades universitarias comienzan a reconocer la importancia de desarrollar dichas competencias como forma de cerrar las brechas con el mercado laboral (OEI, 2020).

Dar mayor prioridad a la formación laboral y profesional continua es una oportunidad para las universidades de lberoamérica, especialmente importante en un contexto en el que ha aumentado el porcentaje de estudiantes que proceden de familias de menores ingresos, las que han sufrido desproporcionadamente los efectos de la crisis de la pandemia. Dadas las bajas tasas de graduación de los estudios universitarios, de alrededor de 40\% en promedio (Ferreyra et al. 2017), es necesaria una innovación que permita habilitar a los estudiantes para empleos productivos, en carreras más cortas, que permitan acceso a empleos en los que existe demanda. Poder atender a esta creciente demanda estudiantil requerirá modalidades programáticas nuevas, más flexibles, menos costosas, y seguramente a distancia.

En América Latina existe un interés creciente por la educación a distancia a nivel superior, en parte por la enorme demanda insatisfecha de acceso a la educación superior, y por la necesidad de modalidades de educación flexibles que permitan acceso a estudiantes en diversidad de condiciones y lugares de residencia. El número de estudiantes cursando el primer título de educación superior a distancia pasó de 2.3 millones en 2010 a 4.5 millones en 2018, representando un $20 \%$ del total de la matrícula (Unesco, 2020). El potencial de utilizar la educación a distancia para fortalecer las capacidades humanas en diversas industrias y en el sector público es enorme.

La necesidad de asimilar el dividendo de innovación pedagógica que haya podido generar la pandemia se deriva, en buena medida, de la aspiración de innovar en materia de enseñanza universitaria, que es una aspiración continua de la universidad, mayor a medida que los cambios tecnológicos, económicos y sociales aumentan las demandas en las competencias que deben tener los egresados universitarios, así como la necesidad de incluir a grupos previamente excluidos y de encontrar modelos financieramente sustentables de hacerlo. Este es el tipo de reinvención de la universidad que ha logrado hacer la Universidad Estatal de Arizona mencionada anteriormente. 
El desarrollar la capacidad de los egresados universitarios para tener impacto en sus sociedades es una de las aspiraciones que guía esta búsqueda de nuevas formas pedagógicas. Ella está en la base de la creación de programas de formación interdisciplinarios, de creación de laboratorios de innovación y de emprendimiento en los que los estudiantes universitarios aprenden no solo a contemplar la complejidad de desafíos, sino a participar en la creación de soluciones. El aprendizaje basado en proyectos y en problemas es la base de mucha de la experimentación pedagógica universitaria, y los desafíos supercomplejos creados por la pandemia ofrecen numerosas oportunidades de involucrar a los estudiantes, como parte de sus estudios, en la creación de soluciones.

\section{Conclusión}

Es cada vez más evidente que la pandemia de la COVID-19 generó considerables pérdidas educativas en Iberoamérica y en el mundo, así como numerosas otras complicaciones sociales. Es fundamental pasar de la contemplación de dichas pérdidas a la reconstrucción de sistemas educativos más resilientes, que permitan desarrollar las capacidades que serán necesarias para reconstruir mejor.

En esta tarea las universidades tienen un papel fundamental que cumplir. A través de la integración de sus funciones de vinculación social, de investigación y de docencia pueden reinventar su lugar en la sociedad, convirtiéndose en las instituciones que auspicien el renacimiento que será necesario para superar la devastación causada por la pandemia. Para ello, deberán innovar y favorecer la innovación en otras instituciones, incluidos los sistemas educativos. La mayor articulación de las universidades con los sistemas educativos, en aras a construir una educación mejor y más relevante para todos, animada por el objetivo de construir sociedades más incluyentes, resilientes, prósperas y sustentables, es la mejor oportunidad que ha creado la pandemia en el campo de la educación. Aprovechar dicha oportunidad requerirá de un buen liderazgo y de conocimiento, a la altura de la profundidad de la crisis creada por la mayor calamidad educativa en la historia de la humanidad.

\section{Agradecimientos}

Aprecio las sugerencias de Luis Enrique García de Brigard, Eugenia Garduño, Reyes Tamez Guerra, Carlos Iván Moreno, Manuel Palacios, Miguel Ángel Santos, Nieves Segovia y Cecilia María Vélez a un borrador de este artículo.

\section{Referencias}

ANUIES (2021). Vinculación de las IES con el Entorno. Asociación Nacional de Universidades e Instituciones de Educación Superior. Recuperado de https://bit.ly/3ArAbOU

Bassi, M., Busso, M., Urzua, S. y Vargas, J. (2012). Desconectados. Habilidades, Educación y Empleo en America Latina. Washington, DC: Banco Interamericano de Desarrollo.

Constitución de Massachusetts de 1780. Recuperado de https://malegislature.gov/laws/constitution

Cooperrider, D., Whitney, D. \& Stavros, J. (2004). Appreciative Inquiry Handbook for Leaders of Change. Brunswick, OH: Crown Custom Publishing, Inc.

Crow, M. y Dabars, D. (2015). Designing the New American University. Baltimore, MA: Johns Hopkins University Press. 
Fernandez-Lamarra, N. (2012) La Educación Superior en América Latina. Interrogantes y desafíos para el debate. Sociedad y Discurso, 21, 94-113.

Ferreyra, M., Avitabile, C., Botero, J., Haimovich, F. y Urzúa, S. (2017). At a Crossroads: Higher Education in Latin America and the Caribbean. Directions in Development. Washington, DC: World Bank.

Gaviria, A. (2020). Muchas Universidades van a empezar a concebirse a si mismas como plataformas. Entrevista por Juanita León. La Silla Vacía. Recuperado de https://bit.ly/3s49EEp.

G-20 High Level Independent Panel (2021). A Global Deal for Our Pandemic Age. Recuperado de https://pandemic-financing.org/report/foreword/

Goel, V. (2021). Universities have a once-in-a-century chance to reset. What should they look like post-COVID? The Globe and Mail. Recuperado de https://tgam.ca/3yMnRIV

Guterres, A. 2020. Presentación del Informe sobre los efectos del COVID-19 en América Latina y el Caribe. Naciones Unidas. Recuperado de https://bit.ly/2U7ADm6

Huntington, S P. (1993). The third wave: Democratization in the late twentieth century, U. of Oklahoma Press.

Jabonero, M., Iglesias, E. y García, E. (2020). Presentacion. Podium, 7.

OEI (2020). Educación Superior, Productividad y Competitividad en Iberoamérica. Madrid: Organización de Estados Iberoamericanos. Recuperado de https://bit.ly/2VDCCz1

ONU (2020a). Respuesta integral de las Naciones Unidas a la COVID-19: salvar vidas, proteger a las sociedades, recuperarse mejor. Organización de Naciones Unidas. Recuperado de https://bit.ly/37rvUi2.

ONU (2020b). El impacto del COVID-19 en América Latina y el Caribe. Organización de Naciones Unidas.

Racine, K. (2002). Francisco de Miranda, a transatlantic life in the Age of Revolution. Wilmington, Del: SR Books.

Reif, R. (2021) The 'super wicked problem' of climate change is our Earthshot. Boston Globe. April 19, 2021. A8.

Ramirez, F. (2020) The Socially Embedded American University: Intensification and Globalization. In Engwall, L. (Ed.) Missions of Universities. Past, Present and Future (pp. 131-161). Switzerland: Springer.

Reimers, F. (Ed.) (2021a). Primary and Secondary Education During COVID-19. Switzerland: Springer. Recuperado de https://bit.ly/3Aq6Q09

Reimers, F. (2021b). Education and COVID-19. Recovering from the shock created by the pandemic and building back better. Switzerland: International Academy of Education and UNESCOInternational Bureau of Education.

Reimers, F. y Schleicher, A. (2020). Schooling disrupted, schooling rethought. How the COVID-19 Pandemic is changing education. Paris: OECD.

Reimers, F. y Marmolejo, F. (Eds.) (2021). University and School Collaborations during a Pandemic. Switzerland: Springer

Reimers, F. y Opertti, R. (Eds.) (2021). Learning to Build Back Better Futures for Education. Lessons from educational innovations during the COVID-19 Pandemic. UNESCO. International Bureau of Education.

Rios, G. (2020). Educación Superior, Competitividad y Productividad en Iberoamérica. Podium ,7, 4-19. UNESCO (2020). Investigación y vínculo con la sociedad en universidades de América Latina. Paris: UNESCO.

UNESCO (2021). Learning to Become Together. A Report of the International Commission on the Futures of Education. Paris: Unesco.

Cómo citar en APA:

Reimers, F. (2021). ¿Cómo puede la universidad contribuir a construir un futuro mejor durante la pandemia de la COVID-19?. Revista Iberoamericana de Educación, 86(2), 9-28. https://doi.org/10.35362/rie8624690 